\title{
Estimación del alcance de radiotransmisores Xbee
}

\author{
José Cruz Núñez Pérez ${ }^{1}$, Aldo Bonilla Rodriguez², Andrés Calvillo Téllez ${ }^{1}$ \\ ${ }^{1}$ Instituto Politécnico Nacional, CITEDI, Baja California, \\ México \\ ${ }^{2}$ Instituto Politécnico Nacional, IPN-UPIITA, Ciudad de México, \\ México
}

nunez@citedi.mx, calvillo@citedi.mx, aldo.bonilla.r@gmail.com

\begin{abstract}
Resumen. Este artículo trata dos aspectos importantes de la comunicación de redes inalámbricas, el primero estima el máximo alcance del enlace para operar dentro del estándar XBee, en la frecuencia de $2.4 \mathrm{GHz}$ que fija la Potencia Isotrópica Radiada Equivalente a $20 \mathrm{dBm}$, y el segundo, estima el alcance máximo que se puede obtener. Considerando los parámetros de calidad del enlace como potencia radiada, ganancia de antenas, atenuación por propagación en espacio libre, margen de desvanecimiento y nivel de recepción de la señal. Los resultados obtenidos muestran en la simulación en Matlab la gráfica referente a: la relación que existe entre la suma de Ganancias Vs distancia de propagación y el rango permisible antes de que el nivel de RSSI alcance su valor mínimo de $100 \mathrm{~dB}$.
\end{abstract}

Palabras clave: conectividad inalámbrica, redes de sensores inalámbricos, RSSI.

\section{Estimation of Range of Xbee Radio Transmitters}

\begin{abstract}
This article treats two important aspects of the communication of wireless networks, the first one estimates the maximum scope of the link to operate inside the standard XBee, in the frequency of $2.4 \mathrm{GHz}$ that fixes the Equivalent Isotropic Radiated Power at $20 \mathrm{dBm}$, and the second one estimates the maximum scope that can be obtained. Considering the quality parameters of the radiolink to be removed, power, profit of antennas, attenuation for a spread in free space and the margin of faint and level of receipt of the signal. The obtained results show in the simulation in Matlab the graph relating to the relation that exists between the sum of Earnings Vs distances of spread and the maximum range before RSSI's level reaches his minimal value of-100dB.
\end{abstract}

Keywords: RSSI, wireless connectivity, wireless sensor networks. 


\section{Introducción}

\subsection{Antecedentes}

Existen un número creciente de servicios y aplicaciones de redes inalámbricas que requieren del uso del espectro electromagnético reservado para las áreas industrial, científica y médica (ISBN, por sus siglas en inglés), que utilizan la banda de frecuencias comprendida entre $2,4 \mathrm{GHz}$ y $2,5 \mathrm{GHz}$. Los radios XBee hacen uso de esta banda y proporcionan conectividad inalámbrica de punto a punto o multipunto para aplicaciones de redes de malla. Utilizan el protocolo de red IEEE 802.15.4 para dispositivos de corto alcance, están diseñados para aplicaciones de alto rendimiento que requieren baja latencia y tiempo de comunicación predecible. Estos dispositivos, cuentan con dos tipos de radiocomunicación, para enlaces punto a punto o bien enlaces de redes malla; la serie 1, emplea un microchip de Freescale ampliamente usado en radiocomunicación punto a punto y la serie 2 de Ember Networks basadas en la creación de redes de malla bajo el protocolo Zig Bee. Ambos radiotransmisores poseen una gama de potencias de transmisión que depende de la máxima distancia que logre la cobertura. Sin embargo, estos dispositivos, presentan limitaciones en cuanto a que hay pocas opciones de modificación de los parámetros de potencia de transmisión, sensibilidad de recepción, ganancia de transmisión y recepción, por lo que hay que trabajar con estas restricciones.

Con el PIRE fijo a máxima potencia de $20 \mathrm{dBm}$ y ganancias comunes en las antenas de transmisión y recepción de $1.5 \mathrm{~dB}$, estos valores influyen en la restricción de la máxima distancia a la cual pueden comunicarse sin que presente perdidas en los datos. El canal de radio inalámbrico es susceptible a interferencias lo que lo hace un medio de comunicación presente fluctuaciones en la potencia de la señal recibida, que disminuye su fiabilidad en la medida en que se incrementa la distancia y aumenta la taza de transmisión [1-3]. Estas perturbaciones modifican su intensidad durante el día, provocada por los mecanismos de propagación y los fenómenos físicos que afectan a las ondas electromagnéticas, a través de la absorción, reflexión, dispersión y difracción.

Para determinar el alcance de comunicaciones fiables, es necesario interpretar el parámetro de indicador de fuerza de la señal recibida (RSSI, por sus siglas en inglés). La escala que presenta emplea como el valor del alcance (d), referido a una distancia de un metro ( $\mathrm{d} 0)$ y una radiación de un $\mathrm{mW}$, para medir el nivel de potencia recibida por un dispositivo inalámbrico a una distancia $(\mathrm{d})$ expresada en metros. La escala inicia en cero, como punto de radiación de la potencia isotrópica efectivamente radiada centro; representa $0 \mathrm{RSSI}$, o $0 \mathrm{dBm}$. Aunque teóricamente puede darse el caso de medirse valores positivos, generalmente la escala se expresa dentro de valores negativos; cuanto más negativo, mayor pérdida de señal [4-8]. La fig.1 se muestra a la señal RSSI como la potencia útil de las ondas de radio, expresado en decibelios donde $0 \mathrm{~dB}$ representa la señal PIRE en su máxima intensidad y $-120 \mathrm{~dB}$ representa la señal cercana al umbral de recepción o el piso de ruido del módulo receptor. Los valores menos negativos representan una señal más limpia o poco corrompida por los mecanismos que imponen los fenómenos físicos de atenuación. Para las comunicaciones inalámbricas de datos, rango normal es de $-45 \mathrm{~dB}$ a $-87 \mathrm{~dB}$. Cualquier 


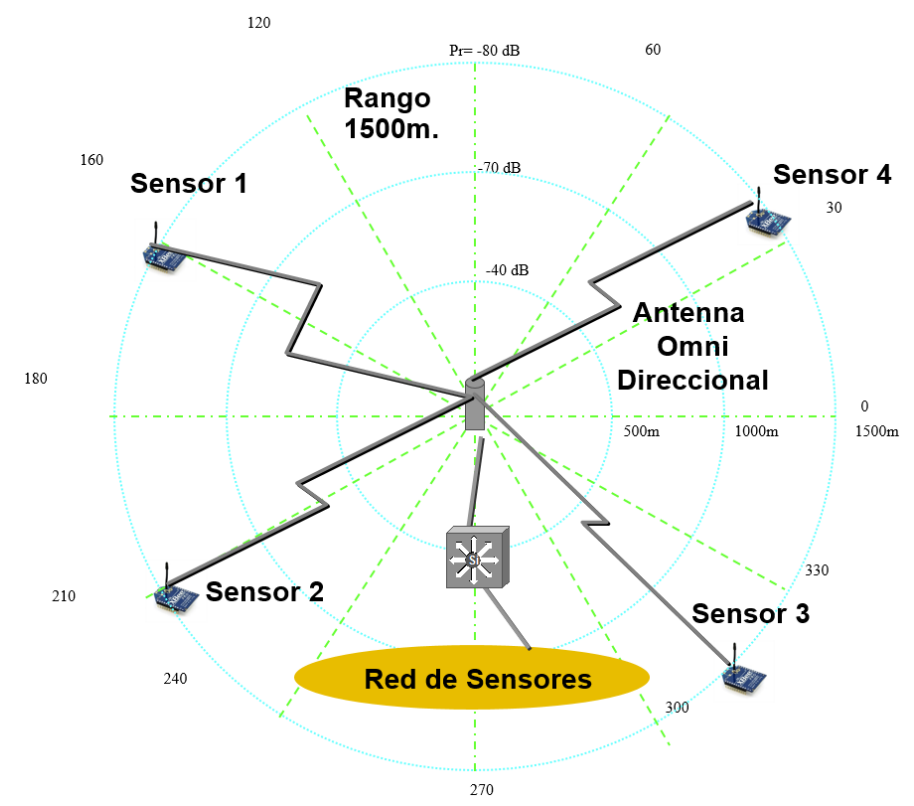

Fig. 1. Rango de la señal, y atenuación respecto al nodo.

Tabla 1. Valores recomendados de RSSI.

\begin{tabular}{ll}
\hline Rango de RSSI & Calidad de la Señal \\
\hline Mejor a $-40 \mathrm{~dB}$ & Excepcional \\
\hline$-40 \mathrm{~dB}$ a $-55 \mathrm{~dB}$ & Muy Bueno \\
\hline$-55 \mathrm{~dB}$ a $-70 \mathrm{~dB}$ & Bueno \\
\hline$-70 \mathrm{~dB}$ a $-80 \mathrm{~dB}$ & Marginal \\
\hline menores a $-80 \mathrm{~dB}$ & Intermitente o no operacional \\
\hline
\end{tabular}

cosa por debajo de $-85 \mathrm{~dB}$ es generalmente inutilizable, y más de $50 \mathrm{~dB}$ puede ser considerado perfecto ver la tabla 1 .

\section{Desarrollo}

Para estimar la distancia de alcance desde que la señal viaja del trasmisor hasta que arriba al receptor. Se analiza la variación de potencia de la señal recibida con respecto a la distancia debido a la disminución de intensidad provocada por los fenómenos físicos que se producen dentro de la trayectoria, causado disipación de la potencia 


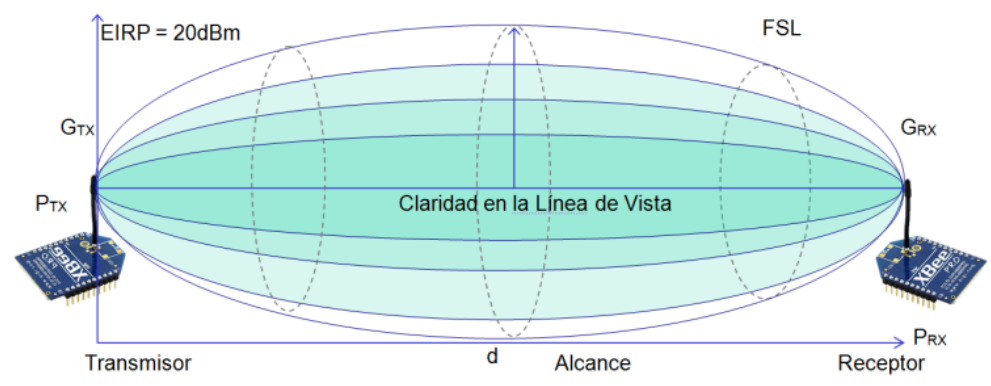

Fig. 2. Parámetros del modelo de Friis.

radiada por el transmisor. En este cálculo se involucran los parámetros que se observan en la figura 2 y cuyo modelo parte de la ecuación de Friis [9-12].

Las ecuaciones de la uno a la ocho relacionan los parámetros de: potencia del transmisor $\mathrm{P}_{\mathrm{TX}}[\mathrm{dBmW}]$, potencia que arriba al receptor $\mathrm{P}_{\mathrm{RX}}[\mathrm{dBmW}]$, después de recorrer la distancia $\mathrm{d}[\mathrm{m}]$, atenuada por las pérdidas de propagación en el espacio libre PPE $[\mathrm{dB}]$, y las ganancias de la antena transmisora $\mathrm{G}_{\mathrm{TX}}[\mathrm{dB}]$ y receptora $\mathrm{G}_{\mathrm{RX}}[\mathrm{dB}]$.

La estimación de la distancia entre los nodos se obtiene a partir de la intensidad de la potencia de la señal recibida que arriba a la antena del módulo receptor:

$$
P P E=10 \log \left(\frac{4 \pi \times 10^{12}}{3 \times 10^{8}}\right)+20 \log d_{k m}+20 \log f_{G H_{z}} .
$$

$P P E_{d B}=$ Perdidas por Propagación en el Espacio en dB.

$d_{k m}=$ Distancia del enlace en $\mathrm{km}$.

$f_{G H z}=$ Frecuencia de operación en $\mathrm{GHz}$.

$$
P I R E=P_{T x}-L_{L T}+G_{T x}
$$

$P I R E_{d B}=$ Potencia Isotrópica Radiada Equivalente en $\mathrm{dB}$.

$P_{T x}=$ Potencia de transmisión en $\mathrm{dB}$,

$G_{R x}=$ Ganancia de la antena de recepción,

$L_{L T}=$ Perdida por conector y por línea de transmisión en $\mathrm{dB}$.

$$
N R S=P I R E-P P E+G_{R x}-L_{L R} .
$$

NRS = Nivel de Recepción de la Señal en dB,

$G_{R x}=$ Ganancia de la antena receptora,

$L_{L R}=$ Perdida por conector y por línea de receptor en $\mathrm{dB}$. 
Estimación del alcance de radiotransmisores Xbee

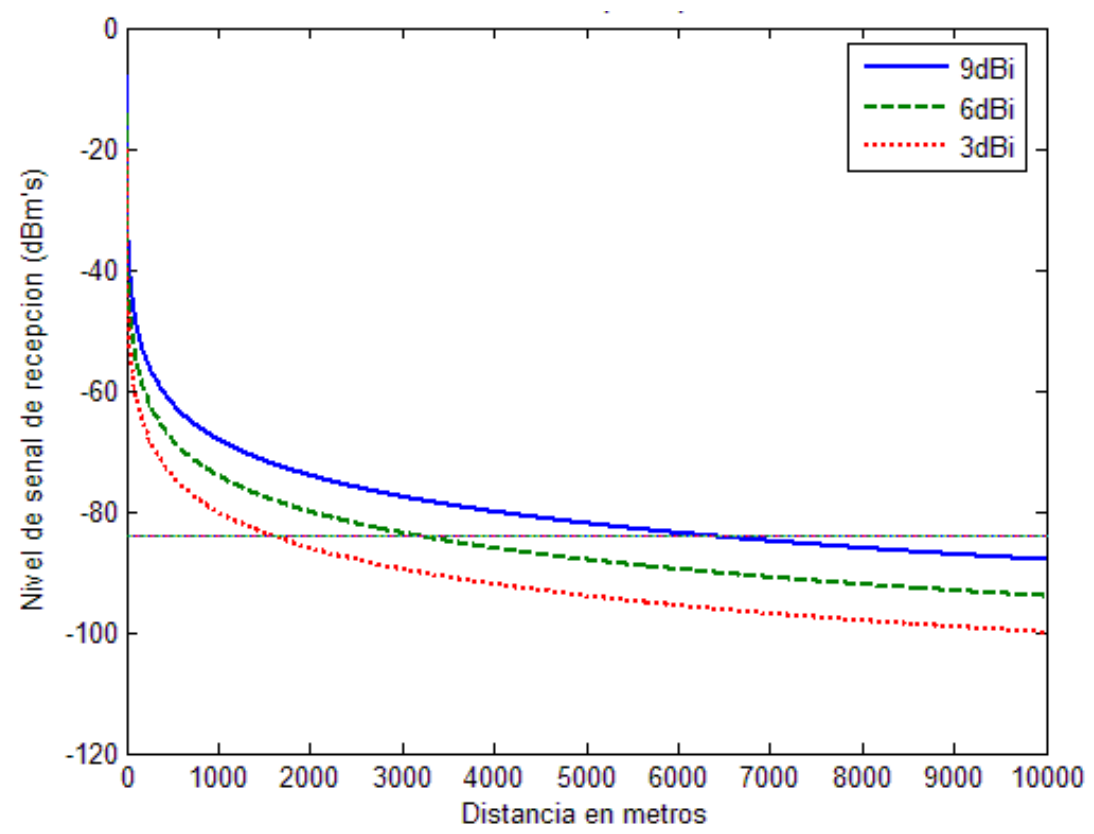

Fig. 3. Relación Distancia vs. Nivel de recepción para frecuencia de $2.4 \mathrm{GHz}$.

$$
M=S-N R S .
$$

$\boldsymbol{M}=$ Margen de desvanecimiento de la Señal, $S=$ Ganancia de la antena receptora.

$$
R S S I=10 \log \left(\frac{N R S}{P_{R e f}}\right)
$$

$N R S=$ Nivel de Recepción de la Señal en mW,

$P_{\operatorname{Re} f}=1 \mathrm{~mW}$.

$$
R S S I=N R S-10 \log (1 \mathrm{~mW}) .
$$

$N R S=$ Nivel de Recepción de la Señal en dB:

$$
P_{R x}=R S S I+M D P_{R x_{\text {Superior }}} \text { si RSSI }>0 .
$$

$M D P_{R x}=$ Margen Dorado de la potencia de Recepción:

$$
P_{R x}=R S S I+M D P_{R x_{\text {Inferior }}} \text { si } R S S I<0 .
$$



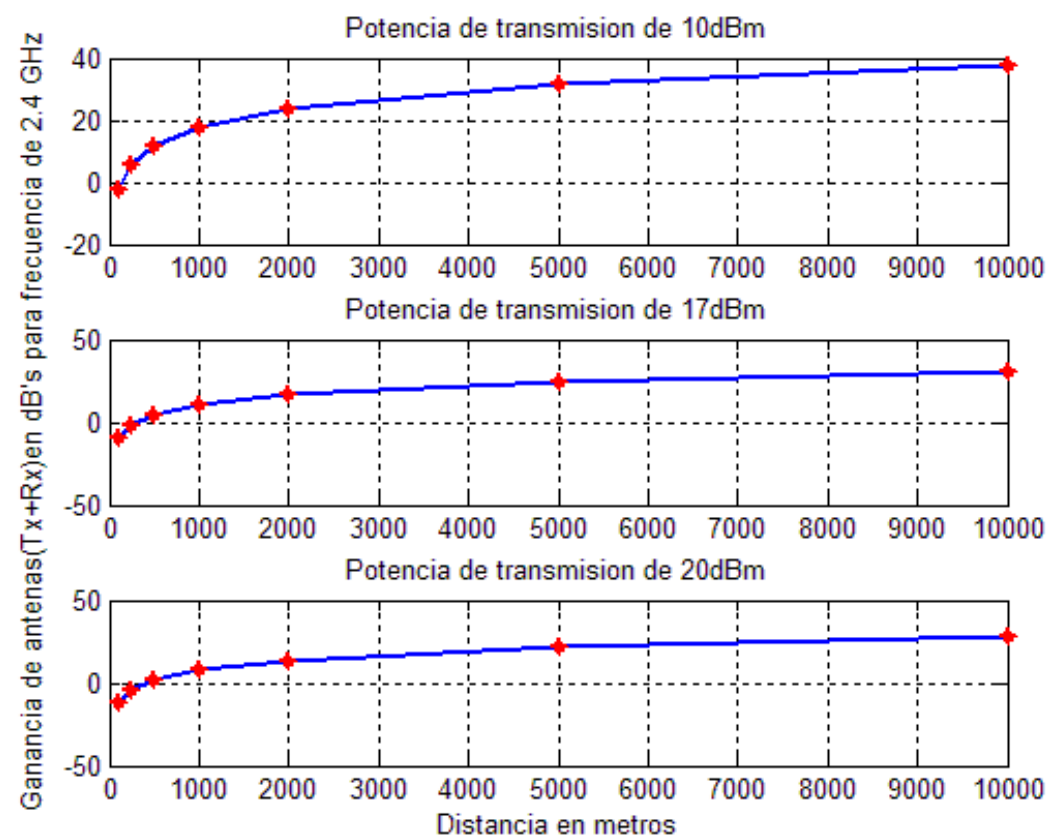

Fig. 4. Relación Distancia vs. Nivel de recepción para frecuencia de 2.4 GHz.

Tabla 2. Valores Medidos de RSSI.

\begin{tabular}{ccccc}
\hline Latitud & Longitud & Descripción & Paquetes 0K & RSSI \\
\hline $32^{0} 32^{\prime \prime} 47^{\prime}$ & $117^{0} 05^{\prime \prime} 4^{\prime}$ & $30 \mathrm{~m}$ & $99 \%$ & $-42 \mathrm{dBm}$ \\
\hline $32^{\circ} 32^{\prime \prime} 47^{\prime}$ & $117^{0} 05^{\prime \prime} 2^{\prime}$ & $100 \mathrm{~m}$ & $99 \%$ & $-64 \mathrm{dBm}$ \\
\hline $32^{\circ} 32^{\prime \prime} 47^{\prime}$ & $117^{0} 04^{\prime \prime} 55^{\prime}$ & $300 \mathrm{~m}$ & $99 \%$ & $-80 \mathrm{dBm}$ \\
\hline $32^{\circ} 32^{\prime \prime} 47^{\prime}$ & $117^{\circ} 04^{\prime \prime} 49^{\prime}$ & $500 \mathrm{~m}$ & $98 \%$ & $-94 \mathrm{dBm}$ \\
\hline $32^{\circ} 32^{\prime \prime} 47^{\prime}$ & $117^{0} 04^{\prime \prime} 39^{\prime}$ & $800 \mathrm{~m}$ & $0 \%$ & - \\
\hline $32^{\circ} 32^{\prime \prime} 47^{\prime}$ & $117^{\circ} 04^{\prime \prime} 33^{\prime}$ & $1000 \mathrm{~m}$ & $0 \%$ & - \\
\hline $32^{\circ} 32^{\prime \prime} 47^{\prime}$ & $117^{\circ} 04^{\prime \prime} 27^{\prime}$ & $1200 \mathrm{~m}$ & $0 \%$ & - \\
\hline $32^{\circ} 32^{\prime \prime} 47^{\prime}$ & $117^{\circ} 04^{\prime \prime} 17^{\prime}$ & $1500 \mathrm{~m}$ & $0 \%$ & - \\
\hline
\end{tabular}

$$
M D P_{R x_{\text {Inferior }}}<P_{R x}+M D P_{R x_{\text {Superior }}} \text { si } R S S I=0 .
$$




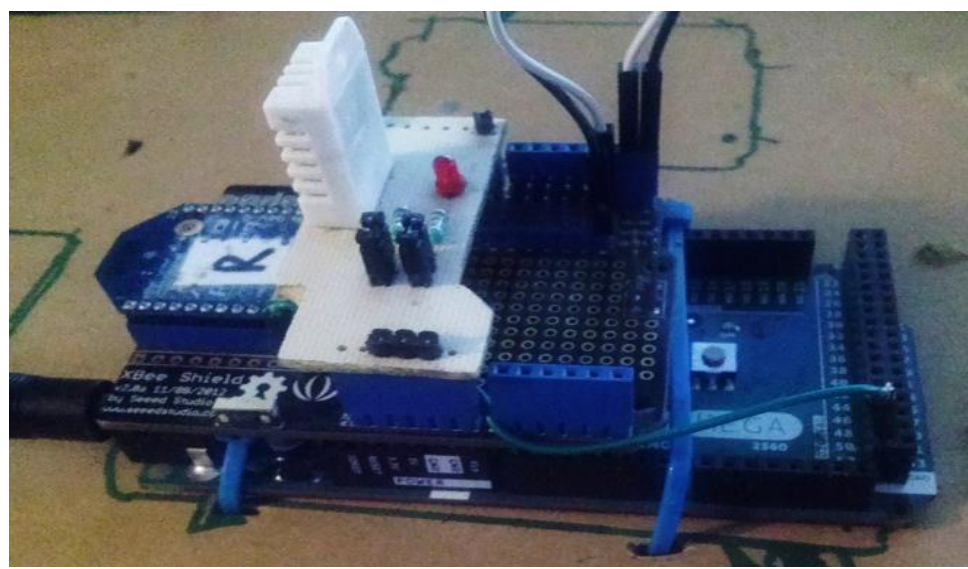

Fig. 5. Módulo de la red de sensores inalámbrica.

\section{Resultados}

Los resultados obtenidos del rango que puede lograr un módulo Xbee en la frecuencia libre de $2.4 \mathrm{GHz}$, empleando la potencia de radiación presente en la antena se fijó a PIRE $=20 \mathrm{dBm}$. A partir del EIRP los alcances máximos se logran con dos elementos el primero lo define el arreglo de antenas para transmitir y recibir [13]; el segundo mediante el análisis de las simulaciones presentadas en las gráficas de las fig. 3 y fig. 4, las cuales muestran las curvas de máximo alcance. Por lo que el elemento de mayor importancia es el umbral de recepción. En la fig. 3 la línea puntuada nos muestra la sensibilidad del receptor XBee, y las demás líneas muestran que teóricamente con una antena de $3 \mathrm{~dB}$ se alcanzaría un rango cercano a los 100 metros, siempre y cuando esté libre de obstáculos, así una antena de 6 dB teóricamente alcanzaría los 3000 metros, y una de $9 \mathrm{~dB}$, puede alcanzar los 6000 metros.

En la fig. 4 se puede observar que para una potencia de transmisión de $10 \mathrm{dBm}$ el rango máximo depende del factor $\mathrm{G}_{\mathrm{Tx}}$ y $\mathrm{G}_{\mathrm{Rx}}$ para un par de antenas con ganancia de 1 $\mathrm{dB}$, la primera marca distancia máxima de separación entre antenas de es de $10 \mathrm{~m}$ la segunda marca indica $10 \mathrm{~m}$ a $1.3 \mathrm{~dB}$ la de $100 \mathrm{~m}$, requiere de antenas de $9 \mathrm{~dB}$, y para la marca de $5000 \mathrm{~m}$ se requiere de una ganancia conjunta cercana a los $36 \mathrm{~dB}$; si las antenas son semejantes para este enlace se tiene que las ganancias de dichas antenas son de $\mathrm{G}_{\mathrm{Tx}}=\mathrm{G}_{\mathrm{Rx}}=18 \mathrm{~dB}$. Y para esta marca de $5000 \mathrm{~m}$ la potencia de transmisión de $17 \mathrm{dBm}$ y $20 \mathrm{dBm}$ se requiere de antenas de $15 \mathrm{~dB}$ y $12 \mathrm{~dB}$ [14-17].

\section{Conclusiones}

Para mantener la comunicación de los nodos en un nivel sin pérdidas de datos, la señal de recepción debe alcanzar valores que no decaigan más allá de los $-70 \mathrm{dBm}$. 
En la medida que se incremente la distancia, la señal recibida disminuye. Si se mejora el sistema de ganancia permitirá mejorar el margen de seguridad del radioenlace. La imagen 3 presenta las máximas distancias que se pueden obtener mediante la ganancia conjunta de las antenas de transmisión y recepción. Es necesario considerar esta ganancia debido a que no siempre se puede obtener el máximo alcance, cuando no se considera esta ganancia es probable que se entre en la violación de la norma pues si consideramos una ganancia superior a $20 \mathrm{dBm}$, ya se está rebasando la recomendación de la norma y se estarán infringiendo las regulaciones de enlaces inalámbricos en banda libre.

Las mediciones permitieron corroborar cómo el RSSI cambia a lo largo de la distancia, las lecturas de la potencia de recepción estuvieron dentro de margen dorado de la potencia de recepción hasta $-50 \mathrm{dBm}$, sin embargo, los valores cercanos al margen dorado de la potencia de recepción inferior, hacían al enlace inestable y con pérdida de datos y por el contrario las lecturas cercanas al margen dorado de la potencia de recepción superior presentaban un enlace robusto.

\section{Referencias}

1. Freeman, R. L.: Telecommunication system engineering. John Wiley \& Sons (2015)

2. Callaway, E. H.: Wireless Sensor Networks. Segunda ed., USA, Prentice Hall (2013)

3. Dorottya, V., Zoltán, V., Rolland, V., Attila, V.: Energy Efficiency in Wireless Sensor Networks Using Mobile Base Station. IFIP International Federation for Information Processing, 196, pp. 173-186 (2006)

4. XBee: XBee-PRO OEM RF Module Antenna Considerations. Application Note XSTAN019, http://ftp1.digi.com/support/images/XST-AN019a_XBeeAntennas.pdf (2015)

5. Freeman, R. L.: Radio System Design for Telecommunications, Wiley \& Sons, (2006)

6. Parson, J. D.: The Mobile Radio Propagation Channel, Wiley \& Sons (1992)

7. Doble, J.: Introduction to Radio Propagation for Fixed and Mobile. Artech House (1996)

8. Bertoni, H. L., et al.: UHF Propagation Prediction for Wireless Personal Communications. Proceedings of the IEEE, 82(9), pp. 1333-1359 (1994)

9. Andersen, J. B., Rappaport, T. S., Yoshida, S.: Propagation Measurements and Models for Wireless Communications Channels. IEEE Communications Magazine, pp. 42-49 (1995)

10. Lee, W. C. Y.: Mobile Communications Design Fundamentals. Second Edition, Wiley \& Sons (1993)

11. CCIR (now ITU-R): Propagation data and prediction methods for the terrestrial land mobile service using the frequency range $30 \mathrm{MHz}$ to $3 \mathrm{GHz}$. Report 567-4, International Telecommunication Union (1990)

12. Gschwender, A.: ZigBee Wireless Sensor and Control Network. Prentice Hall (2009)

13. Figueroa, T. C., Medina, M. J. L., Chávez, P. R. A., Calvillo, T. A.: Circular monopole antenna with defected ground plane for UWB applications. Research in Computing Science, 64, pp. 207-214 (2014)

14. Agrawal, S., Singh, S.: Indoor Localization based on Bluetooth Technology: A Brief Review. International Journal of Computer Applications, ISO 690, 97(8), pp. 31-33 (2014)

15. Gharghan, S. K., Nordin, R., Ismail, M.: Energy-efficient ZigBee-based wireless sensor network for track bicycle performance monitoring. Sensors, ISO 690, 14(8), pp. 15573$15592(2014)$ 